\title{
Demyelination After Primary Central Nervous System Lymphoma; Reversed 'Sentinel'
}

\author{
Barbara Barun, Sandra Kinda, Igor Aurer, Kamelija Žarković, Ivan Adamec, Mario Habek
}

Can J Neurol Sci. 2012; 39: 843-844

A 50-year-old, previously healthy woman presented with memory impairment and handwriting changes. Brain magnetic resonance imaging (MRI) showed hyperintense lesions in T2 and fluid-attenuated inversion recovery (FLAIR) sequences in the left thalamus (Figure 1A and B). Diffuse large B-cell lymphoma was found in a specimen obtained by stereotactic brain biopsy. There were no signs of extraneural lymphoma dissemination. A diagnosis of primary central nervous system (CNS) Large B-cell lymphoma (primary central nervous system lymphoma (PCNSL)) was made. The patient was treated with high-dose methotrexate and whole brain irradiation with completed resolution of clinical symptoms and radiological findings.

Two years later she developed headaches accompanied by dysgeusia, olfactory hallucinations and light-headedness. Brain MRI showed one hyperintense lesion of the right temporal lobe in T2 and FLAIR sequences with homogenous contrast enhancement surrounded with perifocal edema (Figure 1C and D) consistent with relapsed lymphoma. To confirm the relapse a brain biopsy was performed which showed findings consistent with acute disseminated encephalomyelitis (ADEM) (Figure 2). The patient was admitted to our department for further assessment. At that time her neurological examination was unremarkable and her symptoms ameliorated without receiving corticosteroid treatment. Also, the lesion in the right temporal lobe appeared significantly smaller and enhancement was not observed on repeated MRI. Cerebral spinal fluid (CSF) analysis showed 8 cells $/ \mathrm{mm}^{3}$, predominately small lymphocytes; there were no oligoclonal IgG bands. Cytological and microbiological CSF analyses were unremarkable. Extensive work-up to exclude secondary demyelinating lesion was performed including immunological evaluation, HIV, hepatitis, Toxoplasma gondii, Treponema pallidum, Borrelia burgorferi, EBV and CMV serology, angiotensin-convertase and beta2-microglobulin levels. All findings were normal. She was diagnosed as having ADEM and not treated with corticosteroids. Follow-up MRIs, three and six months later, showed no lesions in the right temporal lobe. As well, cognitive evaluation on follow-up was normal. Twelve months later the patient is in remission without symptoms.

\section{Discussion}

We describe a rare association of demyelinating lesion with histological characteristics of ADEM developing two years after PCNSL and suggest a term, reversed "sentinel", for this association. Primary central nervous system lymphoma is a rare malignant tumor accounting for $4 \%$ of all intracranial neoplasms ${ }^{1}$. Contrast-enhanced brain MRI is the standard

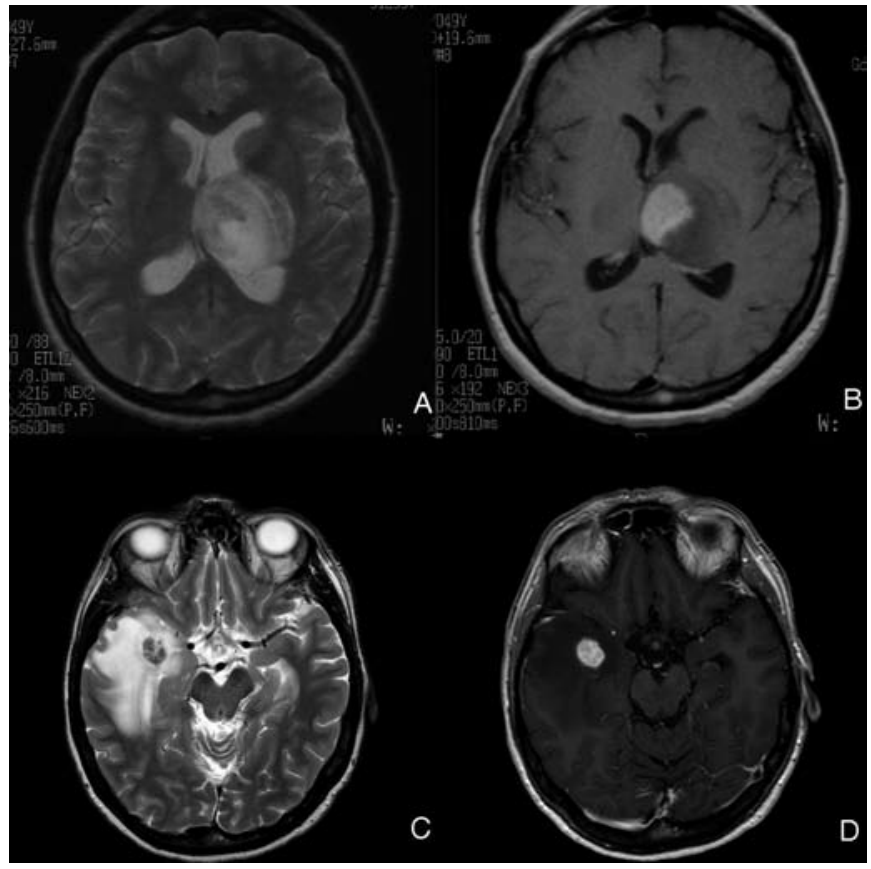

Figure 1: MRI at initial presentation. A) T2-weighted axial image showing hyperintense expansive lesion in the left thalamus, B) T1weighted axial image showing contrast enhancement of the lesion. MRI at suspected PCNSL relapse C) T2- weighted axial showing hyperintense lesion in the right temporal lobe with surrounding edema, D) T1weighted axial image showing contrast enhancement in the right temporal lobe.

neuroimaging method but, to distinguish PCNSL from other potential causes and make a definitive diagnosis, brain biopsy is required. The MRI features of newly diagnosed PCNSL have been described extensively, but there is only one paper describing MRI characteristics at relapse $^{2}$. Recurrence of PCNSL is suggested by newly developing enhancing parenchymal lesions detected by contrast-enhanced T1-weighted

From the Department of Neurology (BB, IAd, MH), Refferal Center for Demyelinating Diseases of the Central Nervous System, Division of Hematology (SK, IAu),

Department of Internal Medicine, Department of Pathology and Cytology (KZ), University Hospital Center Zagreb, Zagreb, Croatia.

Received May 22, 2012. Final Revisions Submitted June 25, 2012 Correspondence to: Mario Habek, University Department of Neurology, University Hospital Center Zagreb, Kišpatićeva 12, HR-10000 Zagreb, Croatia. Email: mhabek@mef.hr. 


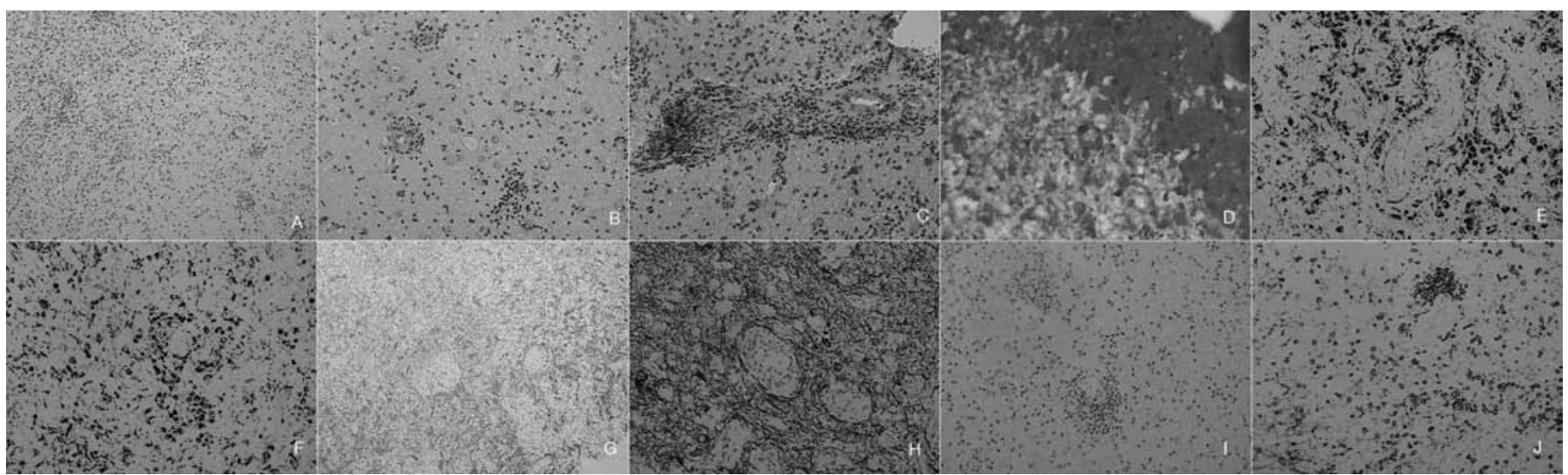

Figure 2: A (magnification 200x), B and C (magnification 400x)) Haematoxylin and eosin section showing predominantly perivascular inflammatory lesions composed predominantly of CD3. J (magnification 400x)) and CD68. E and F (magnification 400x)) positive, and only some CD20 positive B lymphocytes I (magnification 400x)). At the edge of the inflammatory lesion were numerous reactive astrocytes $G$ (magnification $200 x)$ ). NF positive axons were relatively preserved $H$ (magnification 400x)). Kluver-Barrera staining showed loss of myelin D (magnification 400x)). On figure B) a cortical involvement is evident.

MRI-scan in comparison to previous scans, which was the case in our patient. Authors of this study have shown that parenchymal contrast enhancement is the most frequently found MRI sign in PCNSL relapse ${ }^{2}$. However, local recurrence at the site of the initial tumor presentation was only found in $25 \%$ of patients, while in two out of 16 cases, recurrence appeared solely on the contralateral side without involvement of primary lymphoma location ${ }^{2}$. Despite this, PCNSL relapses are sometimes treated based on MRI findings, without histological proof.

Several case reports described demyelinating pseudotumoral lesions preceding PCNSL ${ }^{3,4}$. All of these, so called, 'sentinel' demyelinating lesions occurred prior to PCNSL and receded spontaneously or after corticosteroid treatment. However in many of these case reports patients received corticosteroids prior to biopsy that can make the histological diagnosis of lymphoma difficult ${ }^{5}$. As well perivascular lymphocytic cuffing may be seen in both primary CNS lymphoma and demyelinating lesions. In addition, PCNSL often shows many CD3-positive reactive $\mathrm{T}$ cells admixed with the atypical B cells. CD68-macrophages are also often found in the background of many CNS tumors or destructive processes. Despite of these similarities a spontaneous remission and long follow-up argue against PCNSL relapse. A unique feature of our case is the occurrence of demyelinating lesion two years after PCNSL treatment and its resolution without any treatment. We decided not to give any treatment to the patient because all symptoms resolved spontaneously after brain biopsy and there was a significant improvement in the follow-up MRIs.

Although 'sentinel' demyelinating lesion can precede PCNSL, little is known about the pathogenesis of this peculiar coexistence. Some authors speculated that a host immune response to lymphoma or common viral etiological background could potentially play a role in this 'other way around' scenario with demyelinating lesion appearing after $\mathrm{PCNSL}^{4}$. Newly developed lesions in PCNSL patients are primarily considered to be tumor recurrence but can also be caused by radiation-induced changes (early or delayed), delayed methotrexate neurotoxicity ${ }^{6}$, infection or demyelination.
In summary, discrimination between a demyelinating lesion and PCNSL in patients treated for PCNSL with newly developed neurological impairment may be challenging with clinical and imaging methods. Accurate diagnosis is crucial to avoid delayed treatment of recurrent malignant disease and unnecessary administration of toxic therapies, so clinicians should rely on brain biopsy to confirm suspected relapses.

Despite the favorable scenario in this case, high level of clinical and neuroradiological vigilance is warranted and every new brain lesion in PCNSL patients should be considered tumor recurrence until reliably proven otherwise.

\section{Author Contributions}

Study concept and design: Barun and Habek. Acquisition of data: Barun, Kinda, Aurer, Žarković, Adamec and Habek. Analysis and interpretation of data: Barun, Kinda, Aurer, Žarković, Adamec and Habek. Drafting of the manuscript: Barun. Critical revision of the manuscript for important intellectual content: Barun, Kinda, Aurer, Žarković, Adamec and Habek. Administrative, technical, and material support: Barun, Kinda, Aurer, Žarković, Adamec and Habek.

\section{REFERENCES}

1. Ferreri AJ, Abrey LE, Blay JY, et al. Summary statement on primary central nervous system lymphomas. J Clin Oncol. 2003; 21:2407-14.

2. Schulte-Altedorneburg G, Heuser L, Pels H. MRI patterns in recurrence of primary CNS lymphoma in immunocompetent patients. Eur J Radiol. 2011; http://dx.doi.org/10.1016/j.ejrad. 2011.05.028

3. Alarcia R, Ara JR, Marta E, et al. Demyelinating pseudotumoral lesion prior to a primary cerebral lymphoma. Rev Neurol. 2000; 31:955-8.

4. Alderson L, Fetell MR, Sisti M, et al. Sentinel lesions of primary CNS lymphoma. J Neurol Neurosurg Psychiatry. 1996;60: $102-5$.

5. Habek M, Brinar VV, Zarković K, Ozretić D. Is there sentinel demyelination before development of primary CNS lymphoma? J Clin Neurosci. 2008;15:1069-70.

6. Lai R, Abrey LE, Rosenblum MK, DeAngelis LM. Treatmentinduced leukoencephalopathy in primary CNS lymphoma: a clinical and autopsy study. Neurology. 2004;62:451-6. 